\title{
Composite Taus and Higgs Decays
}

\author{
Adrián Carmona* \\ Institute for Theoretical Physics, \\ ETH Zurich, 8093 Zurich, Switzerland \\ E-mail: carmonaditp.phys.ethz.ch \\ Florian Goertz \\ Institute for Theoretical Physics, \\ ETH Zurich, 8093 Zurich, Switzerland \\ E-mail: fgoertz@itp.phys.ethz.ch
}

In this talk, we describe the effects of extended fermion sectors, respecting custodial symmetry, on Higgs production and decay. The resulting protection for the $Z \rightarrow b_{L} \bar{b}_{L}$ and $Z \rightarrow \tau_{R} \bar{\tau}_{R}$ decays allows for potentially interesting signals in Higgs physics, while maintaining the good agreement of the Standard Model with precision tests. The setups can be motivated as the low energy effective theories of the composite Higgs models $\mathrm{MCHM}_{5}$ and $\mathrm{MCHM}_{10}$, where the spectra can be identified with the light custodians present in these theories. We will show that these describe the relevant physics in the fermion sectors in a simplified and transparent way. In contrast to previous studies of composite models, the impact of a realistic lepton sector on the Higgs decays is taken into account.

The European Physical Society Conference on High Energy Physics -EPS-HEP2013

18-24 July 2013

Stockholm, Sweden

${ }^{*}$ Speaker. 


\section{Introduction}

The properties of the Higgs-like particle discovered at the LHC are so far found to be in good agreement with the Standard Model (SM) predictions. On the other hand, there is also still room for sizable deviations, in particular in the decay into bottom quarks, $\tau$ leptons, and photons. Vector-like fermion scenarios offer viable and simple extensions of the SM with an interesting phenomenology for these Higgs decay modes. Especially new leptons respecting custodial symmetry allow to substantially modify the latter two decays, without generating a conflict with precision tests [1].

In the following, we will examine on the one hand the impact of simple and transparent (lowenergy) vector-like fermion scenarios, featuring a custodial symmetry to protect the $Z b_{L} \bar{b}_{L}$ and $Z \tau_{R} \bar{\tau}_{R}$ couplings, on Higgs physics at the LHC. Beyond that, we will however also address the question where these fermions come from and study strongly coupled UV completions of the scenarios, delivered by different minimal composite Higgs models (MCHM) [2].

Due to the fact that in such models there exist fermionic resonances associated to the heavy top quark (and $\tau$ lepton), required by custodial symmetry, that are typically much lighter than the actual scale of these models, $m_{\text {cust }} \ll f$, their low energy theories match just the aforementioned vectorlike fermion setups. Although, due to the smaller masses, there is a priori no reason to expect such light custodians also in the lepton sector, note that explaining the observed pattern of lepton masses and mixings with the help of a discrete $A_{4}$ symmetry requires the $\tau$ to be more composite than naively expected and thus triggers the appearance of light $\tau$ custodians [3]. At low energies $E \ll f$ one would thus see the SM spectrum plus resonances coming with the top quark and the $\tau$ lepton, which just corresponds to what we will be studying. The additional (sub-leading) impact of the UV completions will be detailed at the end, concentrating on the most important effect of the non-linearity of the Higgs-sector. Due to the compositeness of the $\tau$ one expects non-negligible effects in the lepton sector of Higgs physics, which had been neglected in the past literature.

\section{Setup}

The emergence of light $\tau$ custodians within the framework of the full $\mathrm{MCHM}_{5}$ has been motivated in [3] and they persist also when the $\tau_{R}$ partners transform in a 10 of $S O(5)$ [4]. However, as mentioned before, the setup for our analysis of Higgs production and decay will just rely on the corresponding low energy theories, including the light custodians. For arbitrary fermionic representations, this low energy spectrum will not necessarily be a good description of the full theory of gauge Higgss unification (GHU), as the a priori suppressed contributions of heavier modes can be lifted by larger Yukawa couplings. Nevertheless, for the models we will consider here, the full theory is indeed effectively described by the effects of the light custodians [1]. In particular, we will study the cases where the composite partners of the $\tau_{R}$ transform in fundamental or adjoint representations of $S O(5)$, while all other composite fermions spam fundamental representations, which we will denote by $\mathrm{MCHM}_{5}$ and $\mathrm{MCHM}_{5+10}$, even if we only study the low energy setups.

\section{1 $\mathrm{MCHM}_{5}$}

The light $\tau$ custodians present in this model in addition to the SM fermions are contained in the following degenerate lepton doublets [5]

$$
L_{1 L, R}^{(0)}=\left(\begin{array}{c}
N_{1 L, R}^{(0)} \\
E_{1 L, R}^{(0)}
\end{array}\right) \sim \mathbf{2}_{-\frac{1}{2}}, \quad L_{2 L, R}^{(0)}=\left(\begin{array}{c}
E_{2 L, R}^{(0)} \\
Y_{2 L, R}^{(0)}
\end{array}\right) \sim \mathbf{2}_{-\frac{3}{2}},
$$


where we are explicitly giving the quantum numbers under the electroweak gauge group $S U(2)_{L} \times$ $U(1)_{Y}$. The superscript $(0)$ indicates the current basis. The model is designed such that the custodial symmetry can protect the $Z \tau_{R} \bar{\tau}_{R}$ coupling via a $P_{L R}$ symmetry. In addition, we assume a similar embedding of the quark sector [1], with the replacement $N \rightarrow \Lambda, E \rightarrow T$ and $Y \rightarrow B$ and hypercharges $7 / 6$ and $1 / 6$ respectively, now featuring a protection for the $Z b_{L} \bar{b}_{L}$ coupling. Neglecting the first two generations, which are assumed to have negligible couplings to the new resonances, the mass and Yukawa couplings for the lepton sector are given by

$$
\mathscr{L}_{L}=-y_{l} \bar{l}_{L}^{(0)} \varphi \tau_{R}^{(0)}-y_{l}^{\prime}\left[\bar{L}_{1 L}^{(0)} \varphi+\bar{L}_{2 L}^{(0)} \tilde{\varphi}\right] \tau_{R}^{(0)}-M_{l}\left[\bar{L}_{1 L}^{(0)} L_{1 R}^{(0)}+\bar{L}_{2 L}^{(0)} L_{2 R}^{(0)}\right]+\text { h.c. }
$$

where $\varphi$ is the Higgs doublet. A similar expression holds for the quark sector. We have neglected the couplings of the right handed neutrino (bottom quark), as they are SM like since they do not have any new resonance to couple to.

After diagonalizing the different mass matrices, the Higgs couplings with leptons become [1]

$$
g_{h 5}^{E}=U_{L}^{5 \dagger}\left(s_{L}\right) g_{h 5}^{E(0)} U_{R}^{5}\left(s_{R}\right)=\frac{1}{v}\left(\begin{array}{cccc}
c_{R}^{2} m_{\tau} & 0 & s_{R} c_{R} m_{\tau} \\
0 & 0 & 0 \\
s_{R} c_{R} M_{E_{2}} & 0 & s_{R}^{2} M_{E_{2}}
\end{array}\right),
$$

where $m_{\tau}, M_{E_{1}}$, and $M_{E_{2}}$ are the physical $Q=-1$ lepton masses and $s_{L, R}=\sin \left(\theta_{L, R}\right), c_{L, R}=$ $\cos \left(\theta_{L, R}\right)$ are the sine and the cosine of the mixing angles. It turns out that $s_{R}$, measuring the mixing of the $\tau_{R}$ with the resonances, together with one of the $M_{E_{i}}$ can be taken as the only free parameters of the model. Analogous couplings arise for the $T$ sector [1]. Note that the other new resonances present in the lepton and quark sectors do not couple to the Higgs boson at all.

\section{2 $\mathrm{MCHM}_{5+10}$}

In this case, as the composite partners of the $\tau_{R}$ transform in an adjoint representation of $S O(5)$, which decomposes under $S U(2)_{L} \times S U(2)_{R}$ as $\mathbf{1 0}=(\mathbf{2}, \mathbf{2})+(\mathbf{3}, \mathbf{1})+(\mathbf{1}, \mathbf{3})$, the lepton spectrum will have on top of the bidoublet spanned by (2.1) one additional $S U(2)_{L}$ triplet and two singlets

$$
L_{3 L, R}^{(0)}=\left(\begin{array}{c}
N_{3 L, R}^{(0)} \\
E_{3 L, R}^{(0)} \\
Y_{3 L, R}^{(0)}
\end{array}\right) \sim \mathbf{3}_{-1}, \quad N_{2 L, R}^{(0)} \sim \mathbf{1}_{0}, \quad Y_{1 L, R}^{(0)} \sim \mathbf{1}_{-2} .
$$

The relevant part of the Yukawa and mass Lagrangian for this case can be found in [1]. The rotations to the mass basis will be analogous to the previous case but now featuring larger matrices. However, in this case the $Q=-2$ vector-like leptons $Y_{i}$ will have non vanishing Yukawa couplings to the Higgs boson, leading to a very distinct phenomenology compared to the $\mathrm{MCHM}_{5}$.

\section{Higgs Production and Decay}

The presence of the new resonances has interesting implications on the production and decay of the Higgs boson, which will be worked out in this section. We will first concentrate on the $\mathrm{MCHM}_{5}$ and later discuss the generalization to the $\mathrm{MCHM}_{5+10}$.

The most important production mechanism for the Higgs boson at hadron colliders is gluongluon fusion, which in the SM receives its main contribution from a top-quark triangle loop. Here, 
the new quarks (the top custodians) give rise to additional loop diagrams, with contributions proportional to the corresponding Higgs coupling over the new quark masses

$$
v_{T}^{5} \equiv v \sum_{n=2}^{3} \frac{\operatorname{Re}\left[\left(g_{h 5}^{T}\right)_{n n}\right]}{m_{T_{n-1}}} A_{q}^{h}\left(\tau_{T_{n-1}}\right) \approx\left(s_{R}^{t}\right)^{2},
$$

where $\tau_{i}=4 m_{i}^{2} / m_{h}^{2}$. In the second step we have already inserted the explicit result for our model, following from (2.3) and used the fact that the loop function $A_{q}^{h}$ approaches an asymptotic value of 1 for virtual particles much heavier than the Higgs, which is already a good approximation for the SM top quark. For details, see [1]. Moreover, there are changes in the couplings of the Higgs to SM-like quarks, which for the top quark amount to a reduction of

$$
\kappa_{t}^{5} \equiv \nu \operatorname{Re}\left[\left(g_{h 5}^{T}\right)_{11}\right] / m_{t}=\left(c_{R}^{t}\right)^{2},
$$

and vanish for bottom quarks, $\kappa_{b}^{5}=1$.

The deviations in gluon fusion can now be parametrized by a rescaling factor $\kappa_{g}^{5}$ as

$$
\begin{gathered}
\sigma(g g \rightarrow h)_{\mathrm{MCHM}_{5}}=\left|\kappa_{g}^{5}\right|^{2} \sigma(g g \rightarrow h)_{\mathrm{SM}}, \\
\kappa_{g}^{5}=\frac{\sum_{f=t, b} \kappa_{f}^{5} A_{q}^{h}\left(\tau_{f}\right)+v_{T}^{5}}{\sum_{f=t, b} A_{q}^{h}\left(\tau_{f}\right)} \approx \frac{\left(c_{R}^{t}\right)^{2}+A_{q}^{h}\left(\tau_{b}\right)+\left(s_{R}^{t}\right)^{2}}{1+A_{q}^{h}\left(\tau_{b}\right)} .
\end{gathered}
$$

Employing $\left(c_{R}^{t}\right)^{2}+\left(s_{R}^{t}\right)^{2}=1$, we see explicitly that the new loop contributions from the top custodians cancel with the corrections to the SM-like loops and thus gluon fusion remains to leading approximation SM-like, i.e. $\kappa_{g}^{5} \approx 1$. This cancellation of the fermionic parameters agrees with the effects found in composite models [6]. Finally, the change in the $h t \bar{t}$ vertex also modifies $t t h$ production, leading to $\sigma(t t h)_{\mathrm{MCHM}_{5}}=\left(\kappa_{t}^{5}\right)^{2} \sigma(t t h)_{\mathrm{SM}}$, whereas other relevant production mechanisms are unaffected by the new fermions, $\sigma(\mathrm{VBF})_{\mathrm{MCHM}_{5}}=\sigma(\mathrm{VBF})_{\mathrm{SM}}$ and $\sigma(V h)_{\mathrm{MCHM}_{5}}=\sigma(V h)_{\mathrm{SM}}$.

We now turn to the Higgs decays and want to analyze in particular if a similar cancellation that happens in gluon fusion also appears in the loop induced decay into photons. We define

$$
\Gamma(h \rightarrow f f)_{\mathrm{MCHM}_{5}}=\left|\kappa_{f}^{5}\right|^{2} \Gamma(h \rightarrow f f)_{\mathrm{SM}},
$$

$f=\gamma, W, Z, b, \tau, g$. Since we did not change the bosonic sector of the SM so far, we get $\kappa_{W}^{5}=\kappa_{Z}^{5}=$ 1. Beyond that, the rescaling factors for the (tree-level) decays into two fermions as well as the decay to gluons, entering (3.5), have already been specified in (3.2), with obvious replacements for $\kappa_{\tau}^{5}$, and (3.4). Note that in our setup the couplings of the light fermions to the Higgs boson are unchanged. ${ }^{1}$ For the effective coupling to photons we obtain

$$
\begin{aligned}
\kappa_{\gamma}^{5} & =\frac{\sum_{f=t, b} N_{c} Q_{f}^{2} \kappa_{f}^{5} A_{q}^{h}\left(\tau_{f}\right)+Q_{\tau}^{2} \kappa_{\tau}^{5} A_{q}^{h}\left(\tau_{\tau}\right)+A_{W}^{h}\left(\tau_{W}\right)+N_{c} Q_{t}^{2} v_{T}^{5}+Q_{\tau}^{2} v_{E}^{5}}{\sum_{f=t, b} N_{c} Q_{f}^{2} A_{q}^{h}\left(\tau_{f}\right)+Q_{\tau}^{2} A_{q}^{h}\left(\tau_{\tau}\right)+A_{W}^{h}\left(\tau_{W}\right)} \\
& =\frac{N_{c}\left(Q_{t}^{2}+Q_{b}^{2} A_{q}^{h}\left(\tau_{b}\right)\right)+Q_{\tau}^{2}\left(\left(c_{R}^{\tau}\right)^{2} A_{q}^{h}\left(\tau_{\tau}\right)+\left(s_{R}^{\tau}\right)^{2}\right)+A_{W}^{h}\left(\tau_{W}\right)}{N_{c}\left(Q_{t}^{2}+Q_{b}^{2} A_{q}^{h}\left(\tau_{b}\right)\right)+Q_{\tau}^{2} A_{q}^{h}\left(\tau_{\tau}\right)+A_{W}^{h}\left(\tau_{W}\right)} \approx \frac{-5+\left(s_{R}^{\tau}\right)^{2}}{-5},
\end{aligned}
$$

where $N_{c}=3, Q_{t}=2 / 3, Q_{b}=-1 / 3, Q_{\tau}=-1$. The first, second, and third terms in the numerator in the first row describe the effects of virtual SM-type quark, lepton, and $W^{ \pm}$-boson exchange,

\footnotetext{
${ }^{1}$ In (anarchic) composite models, this is a good approximation due to the tiny mixing with the fermionic resonances.
} 

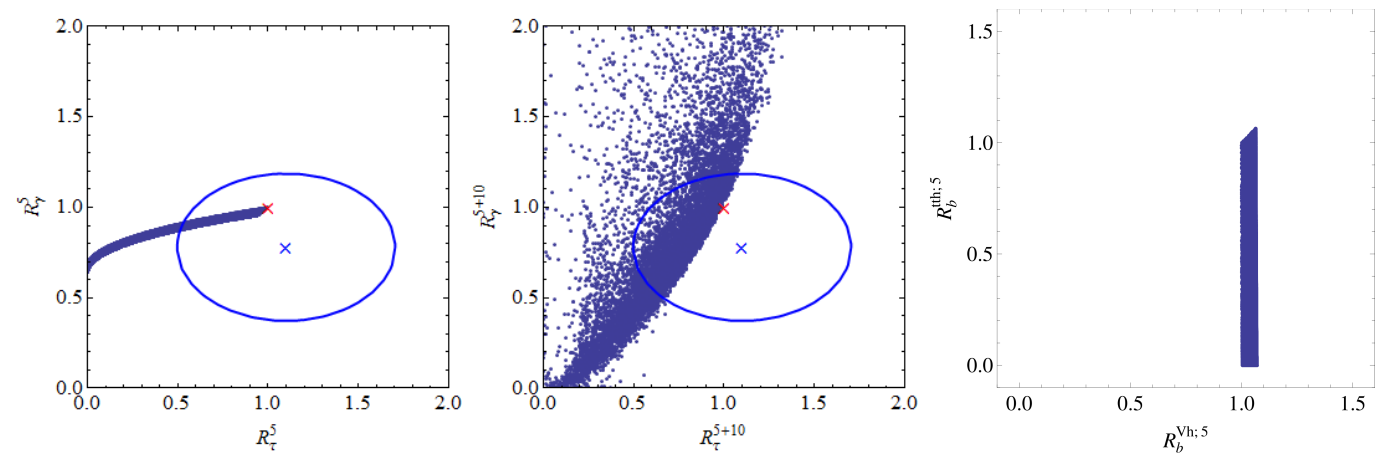

Figure 1: Left and Middle: Production cross section times decay for $p p \rightarrow h \rightarrow \gamma \gamma$ versus $p p \rightarrow h \rightarrow \tau \tau$, relative to the SM, for both models considered. The ellipse sketches the CMS $1 \sigma$ bound. Right: $p p \rightarrow h \rightarrow$ $b b$ via $t$ th production over the SM result versus the same ratio mediated by $V h$. See text and [1] for details.

respectively. The fourth and fifth term, on the other hand, correspond to the contributions of the custodians, which now also include leptonic resonances - the lepton contribution $v_{E}^{5}$ is defined in analogy to (3.1). Note that the amplitude proportional to $A_{W}^{h}\left(\tau_{W}\right) \approx-6.25$ dominates in the SM and interferes destructively with the fermion contributions $A_{q}^{h}\left(\tau_{f}\right)$. In the second row we have already employed that $\left(c_{R}^{t}\right)^{2}+\left(s_{R}^{t}\right)^{2}=1$. One can see an interesting thing happening. Due to the fact that the $\tau$ is much light than the Higgs (leading to $A_{q}^{h}\left(\tau_{\tau}\right) \ll 1$ ) a similar cancellation as for quarks is not happening in the lepton sector, which includes the new case of a light fermion with nevertheless naturally a significant composite component. This leads to a reduction of the Higgs coupling to two photons, which can become quite significant and has been neglected before [1].

In the case of the $\mathrm{MCHM}_{5+10}$, besides a larger parameter space which calls for numerical methods of diagonalization and a random scan to study the phenomenological predictions of the model, we have the distinctive feature of new $Q=-2$ vector-like excitations coupling to the Higgs boson and therefore entering the $h \rightarrow \gamma \gamma$ loop process [1]. On the other hand, as the gauge and quark sectors remain the same, we still have $\kappa_{W}^{5+10}=\kappa_{Z}^{5+10}=\kappa_{b}^{5+10} \approx \kappa_{g}^{5+10} \approx 1$ and $\kappa_{t}^{5+10}=\kappa_{t}^{5}$.

To compare both models and have an idea of the possible size of the deviations with respect to the SM, we show in the left and central panels of Figure 1 the ratio of the production cross section times branching fraction for $p p \rightarrow h \rightarrow \gamma \gamma$ relative to the SM versus the same ratio for $p p \rightarrow h \rightarrow \tau \tau$ in both models (see [1] for more details). While in the $\mathrm{MCHM}_{5}$ we scan $0 \leq s_{R} \leq 1$, in the $\mathrm{MCHM}_{5+10}$ we have assumed random vector-like masses of $\sim(0.2-1) \mathrm{TeV}$ as well as order one Yukawa couplings with the strong sector, motivated from the UV completion. We again refer the reader to [1] for more details. Comparing the plots, we can see that the strong correlations present in the $\mathrm{MCHM}_{5}$ are washed out in the $\mathrm{MCHM}_{5+10}$, making on the one-hand side the model less predictive but on the other allowing in principle for either an excess or a suppression in $p p \rightarrow$ $h \rightarrow \gamma \gamma$. However, in the GHU model completing the $\mathrm{MCHM}_{5+10}$ there are additional correlations present between the parameters. If we impose them to mimic its UV completion, it turns out that all the points corresponding to $R_{\tau}^{5+10}>1$ or $R_{\gamma}^{5+10}>1$ in the middle panel of the previous figure disappear. We have also plotted, in the right panel of Figure 1, the Higgs production cross section in the $t$ th channel times branching fraction $h \rightarrow b b$ relative to the SM versus the equivalent ratio, assuming that the Higgs has been produced via associated vector-boson production. Once the 

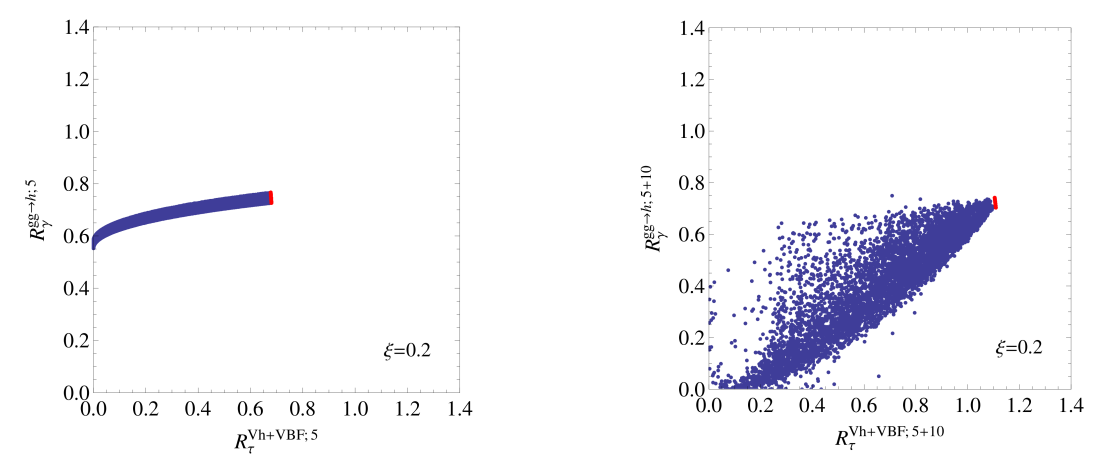

Figure 2: Production cross section times decay for $g g \rightarrow h \rightarrow \gamma \gamma$ relative to the SM versus the same ratio for $h \rightarrow \tau \tau$ in VBF or $V h$ production, including the effects of the non-linearity of the Higgs as well as the additional correlations coming from the UV completion of the $\mathrm{MCHM}_{5+10}$, see text and [1] for more details.

experimental situation in these channels improves they will become a superb tool for measuring directly a possible reduction in the $t$ th coupling.

Up to now, we have neglected the effects arising from the pseudo-Goldstone boson nature of the Higgs in the UV completions of our models. Considering this will lead to shifted Higgs couplings to the different fermions and gauge bosons of the spectrum. In the latter case, neglecting the (sub-leading) mixing of the SM gauge bosons to their composite counterparts, everything is fixed by the quantum numbers and the symmetry breaking defining the composite model [7]

$$
\kappa_{W}^{m}=\kappa_{Z}^{m}=\cos \left(\frac{v}{f}\right) \approx \sqrt{1-\xi}, \quad m=5,5+10,
$$

where we have defined $\xi=v^{2} / f^{2}$ as usual. The rescaling of fermions, on the other hand, depends on the particular representations. In the $\mathrm{MCHM}_{5}$, we have the following universal shift of the Higgs coupling to SM fermions,

$$
\kappa_{f}^{5} \rightarrow \kappa_{f}^{5} \cos \left(\frac{2 v}{f}\right) / \cos \left(\frac{v}{f}\right) \approx \kappa_{f}^{5}(1-2 \xi) / \sqrt{1-\xi}
$$

leading also to a suppression of the effective coupling to gluons,

$$
\kappa_{g}^{m} \approx \cos \left(\frac{2 v}{f}\right) / \cos \left(\frac{v}{f}\right) \approx(1-2 \xi) / \sqrt{1-\xi}, \quad m=5,5+10 .
$$

In the case of the $\mathrm{MCHM}_{5+10}$ we get a different correction for the $\tau$ [8]

$$
\kappa_{\tau}^{5+10} \rightarrow \kappa_{\tau}^{5+10} \cos \left(\frac{v}{f}\right) \approx \kappa_{\tau}^{5+10} \sqrt{1-\xi}
$$

while all other fermionic couplings change analogously as in the $\mathrm{MCHM}_{5}$. We have implemented all these additional corrections in our phenomenological study, employing $\xi=0.2$, to see to what extend the previous picture is changed. To this purpose we show in Figure 2 similar plots to the ones appearing in the left and middle panels of Figure 1, taking into account the effects of the non-linearity of the Higgs (as well as the additional correlations coming from the UV completion, commented on above). Note that in the latter Figure we have also distinguished different production 
mechanisms for the processes considered; namely $g g \rightarrow h$ for $h \rightarrow \gamma \gamma$ and $V h+\mathrm{VBF}$ for $h \rightarrow \tau \tau$. We also show by red regions the predictions corresponding to zero mixing with the composite lepton sector. Comparing these with the effects due to the mixing with the vector-like leptons, we can see that the latter effect is still the dominant one for a composite $\tau$ scenario.

\section{Conclusions and Outlook}

In this note, we have studied the impact of light custodians, present in composite Higgs models, on Higgs production and decay. In particular, we pointed out that the inclusion of a realistic lepton sector can lead to important in effects in the Higgs decays to two photons and $\tau$ leptons, whereas changes in the $t$ th coupling could be probed by comparing different production mechanisms. Due to the effective (low energy) approach of our analyses, it also provides interesting information about general viable vector-like fermion scenarios, independent of the UV completion. Our focus on the third generation can then be motivated by its prominent role in the SM, suggesting a closer connection to electroweak symmetry breaking and new physics. The predicted clear correlations allow for interesting tests of the setups. We presented explicitly the impact of full composite completions, due to the non-linearity of the Higgs sector.

Finally, an obvious question is how composite the $\tau$ can be without generating a conflict with observables that are not protected by custodial symmetry. Tau compositeness can for example lead to sizable effects in $\left(\bar{e} \gamma^{\mu} P_{L, R} e\right)\left(\bar{\tau} \gamma_{\mu} P_{R} \tau\right)$ transitions, due to the exchange of heavy composite resonances. The coefficient of this operator is experimentally constrained to $C_{e e \tau \tau} \lesssim[(1-2) \mathrm{TeV}]^{-2}$ [9]. In the composite models considered here, it is expected to scale as $C_{e e \tau \tau} \sim g^{2} \times 1 / \sqrt{L} \times \sqrt{L} \times$ $\left(s_{R}^{\tau}\right)^{2} / m_{\rho}^{2}$, for $s_{R}^{\tau} \sim \mathscr{O}(1)$, where $L \approx 35$ corresponds to an enhancement/suppression factor of the coupling, depending on the compositeness of the external fermions. Even for such values of $s_{R}^{\tau}$, the bound mentioned before is fulfilled for masses of the bosonic resonances as light as $m_{\rho} \sim \mathscr{O}(1) \mathrm{TeV}$.

Along similar lines, also the one-loop corrections to the $Z \tau \bar{\tau}$ vertex, due to triangles containing fermionic and heavy bosonic resonances, are expected to be modest enough for reasonable regions of the parameter space, such as to still allow for interesting effects in Higgs physics without a conflict with precision tests. A corresponding detailed analysis will be presented elsewhere [4].

Acknowledgements We acknowledge support from the SNF under contract 200021-143781.

\section{References}

[1] A. Carmona and F. Goertz, JHEP 1304 (2013) 163 [arXiv:1301.5856 [hep-ph]].

[2] K. Agashe, R. Contino and A. Pomarol, Nucl. Phys. B 719 (2005) 165 [hep-ph/0412089].

[3] F. del Aguila, A. Carmona and J. Santiago, JHEP 1008 (2010) 127 [arXiv:1001.5151 [hep-ph]].

[4] A. Carmona and F. Goertz, In preparation.

[5] F. del Aguila, A. Carmona and J. Santiago, Phys. Lett. B 695 (2011) 449 [arXiv:1007.4206 [hep-ph]].

[6] A. Falkowski, Phys. Rev. D 77 (2008) 055018 [arXiv:0711.0828 [hep-ph]].

[7] G. F. Giudice, C. Grojean, A. Pomarol and R. Rattazzi, JHEP 0706 (2007) 045 [hep-ph/0703164].

[8] A. Azatov and J. Galloway, Phys. Rev. D 85 (2012) 055013 [arXiv:1110.5646 [hep-ph]].

[9] M. Raidal, A. van der Schaaf, I. Bigi, M. L. Mangano, Y. K. Semertzidis, S. Abel, S. Albino and S. Antusch et al., Eur. Phys. J. C 57 (2008) 13 [arXiv:0801.1826 [hep-ph]]. 